\title{
INVESTIGATION OF INTERVAL STABILITY OF LINEAR SYSTEMS OF NEUTRAL TYPE OF LYAPUNOV FUNCTION METHOD
}

\author{
DENIS YA. KHUSAINOV \\ Kiev University \\ Complex System Simulation Department \\ Vladimirskay 64, 01033 Kiev-33 Ukraine \\ E-mail:denis@dh.cyb.univ.kiev.ua
}

(Received January, 1997; Revised March, 1999)

\begin{abstract}
Systems of differential equations with deviating argument of neutral type [1, 3, 8] are used. The mathematical model takes into account not only the previous moments of time, but also the speed of their change. These equations more adequately describe the dynamics of processes, but their investigation faces significant difficulties. The qualitative behavior of solutions of neutral type systems includes the features both, differential and difference equations [2,9]. Stability investigations require the smallest size of the delay's speed value [7]. Recently, so-called interval, or robust stability theory has received intensive development. It is based on two theorems of V.L. Kharitonov [4,5] for scalar: equations. However, difficulties have appeared to obtain similar results for systems in vector-matrix form. It is even more complicated to derive conditions of interval stability for systems of differential-difference equations, though there are results for scalar equation in $[6,10]$.
\end{abstract}

Key words: Stability, Robust Stability, Systems of Neutral Type, Lyapunov's Functions.

AMS subject classifications: $34 \mathrm{~K} 20$.

\section{Introduction}

In this article, sufficient conditions of interval stability are obtained for systems of differential equations with deviating arguments of neutral type:

$$
\dot{x}(t)=D \dot{x}(t-\tau)+A x(t)+B x(t-\tau) .
$$

Here $D, A, B$ are matrices with constant coefficients and $\tau>0$ is a constant delay. Along with the system (1) we consider an interval system of following form

$$
\dot{x}(t)=(D+\Delta D) \dot{x}(t-\tau)+(A+\Delta A) x(t)+(B+\Delta B) x(t-\tau),
$$

where the elements of the matrices $\Delta D=\left\{\Delta d_{i j}\right\}, \Delta A=\left\{\Delta a_{i j}\right\}, \Delta B=\left\{\Delta b_{i j}\right\}, i, j=\overline{1, n}$ take values from some fixed, symmetric intervals

$$
-\delta_{i j} \leq \Delta d_{i j} \leq \delta_{i j},-\alpha_{i j} \leq \Delta a_{i j} \leq \alpha_{i j},-\beta_{i j} \leq b_{i j} \leq \beta_{i j}, i, j=\overline{1, n} .
$$


We obtain conditions of interval (robust) stability of the system (2), i.e., asymptotic stability with respect to the matrices $\Delta D, \Delta A, \Delta B$.

Definition 1: The solution $x(t) \equiv 0$ of the system (1) is called Lyapunov stable, for arbitrary $\epsilon>0$ there exist $\sigma_{1}>0$ and $\sigma_{2}>0$ such that for any solution $x(t)$ of the system (1), $|x(t)|_{1}<\epsilon$ holds for $t>0$, if $\|x(0)\|_{\tau}<\sigma_{1}$ and $\|\dot{x}(0)\|_{\tau}<\sigma_{1}$.

Definition 2: The solution $x(t) \equiv 0$ of the system (1) is called asymptotically stable if it is Lyapunov stable and $\lim _{t \rightarrow \infty}|x(t)|=0$.

Here and in the following, the vector norms are given by:

$$
\begin{gathered}
|x(t)|=\left\{\sum_{i=1}^{n} x_{i}^{2}(t)\right\}^{1 / 2},|x(t)| 1=\max \{|x(t)|,|\dot{x}(t)|\}, \\
\|x(0)\|_{-\tau}=\max _{-\tau \leq 0}\{|x(s)|\}, \quad\|\dot{x}(0)\|_{\tau-\tau \leq s \leq 0}=\max _{-\tau}\{|\dot{x}(s)|\} .
\end{gathered}
$$

And the matrix norms are given by

$$
|A|=\left\{\lambda_{\max }\left(A A^{T}\right)\right\}^{1 / 2} .
$$

where $\lambda_{\max }(\cdot)$ is the maximum eigenvalue of the corresponding matrix. Let us introduce the following notations:

$$
\begin{gathered}
\|A+\Delta A\|=\max _{\left|\Delta a_{i j}\right| \leq \alpha_{i j}}\{|A+\Delta A|\},\|B+\Delta B\|=\max _{\left|\Delta b_{i j}\right| \leq \beta_{i j}}\{|B+\Delta B|\}, \\
\|D+\Delta D\|=\max _{\left|\Delta d_{i j}\right| \leq \delta_{i j}}\{|D+\Delta D|\},\|\Delta D\|=\max _{\left|\Delta d_{i j}\right| \leq \delta_{i j}}\{|\Delta D|\}, \\
\left\|[(E-D)-\Delta D]^{T} H(B-\Delta B)\right\| d_{i j}=\leq \max _{i j},\left|\Delta b_{i j}\right| \leq \beta_{i j}\left\{\left|[(E-D)-\Delta D]^{T} H(B+\Delta B)\right|\right\}, \\
\left\|[(E-D)-\Delta D]^{T} H(D+\Delta D)\right\|=\max _{\left|d_{i}\right| \leq \delta_{i j}}\left\{\left|[(E-D)-\Delta D]^{T} H(B+\Delta B)\right|\right\}, \\
\left\|(A+B)^{T} H \Delta D-(\Delta A+\Delta B)^{T} H[(E-D)-\Delta D]\right\| \\
\mid \begin{array}{l}
\left|\Delta a_{i j}\right| \leq \alpha_{i j} \\
\left|\Delta b_{i j}\right| \leq \beta_{i j} \\
\left|\Delta d_{i j}\right| \leq \delta_{i j}
\end{array}
\end{gathered}
$$

Definition 3: The system (2) is called asymptotically stable, if its solution $x(t) \equiv 0$ is asymptotically stable.

Definition 4: The system (2) is called interval stable, if it is asymptotically stable for all matrices $\Delta D, \Delta A, \Delta B$ with elements as given in (3).

Herein and after, it is assumed that the "stability" condition for the difference operator, i.e., $\|D+\Delta D\|<1$, is fulfilled and the system without delay

$$
\dot{x}(t)=(E-D)^{-1}(A+B) x(t)
$$


is asymptotically stable. Then there always exist a Lyapunov function of quadratic form, $V(x)=x^{T}(E-D)^{T} H(E-D) x$ where the symmetric, positive definition matrix $H$ is a solution of the Sylvester matrix equation

$$
(A+B)^{T} H(E-D)+(E-D)^{T} H(A+B)=-C
$$

for arbitrary positive definite matrix $C$.

Consider the interval system without delay, i.e., $\tau=0$ :

$$
\dot{x}(t)=[(E-D)-\Delta D]^{-1}[(A+B)+(\Delta A+\Delta B)] x(t) .
$$

We obtain conditions for the interval stability of the system (6) under the assumption that the system (4) is asymptotically stable.

Theorem 1: Let $(E-D)^{-1}(A+B)$ be an asymptotically stable matrix and there exist a symmetric positive definite matrix $H$ such that $L(H)>0$, where

$$
\begin{aligned}
& L(H)=\lambda_{\min }\left[-(A+B)^{T} H(E-D)-(E-D)^{T} H(A+B)\right] \\
& -2\left\|(A+B)^{T} H \Delta D-(\Delta A+\Delta B)^{T} H[(E-D)-\Delta D]\right\|,
\end{aligned}
$$

$\lambda(\cdot)_{\min }, \lambda(\cdot)_{\max }$ are minimum and maximum eigenvalues of corresponding matrices. Then the system (6) is interval stable.

Proof: Take as Lyapunov function the following quadratic form:

$$
V(x)=x^{T}[(E-D)-\Delta D]^{T} H[(E-D)-\Delta D] x
$$

where the symmetric, positive definite matrix $H$ is a solution of the matrix equation (5). For an arbitrary matrix $\Delta D$ satisfying the "stability" condition, this function is positive definite.

Consider the total derivative along solution of (6):

$$
\begin{gathered}
\dot{V}(x(t))=x^{T}(t)\left\{[(A+B)+(\Delta A+\Delta B)]^{T} H[(E-D)-\Delta D]\right. \\
\left.+[(E-D)-\Delta D]^{T} H[(A+B)+(\Delta A+\Delta B)]\right\} x(t) .
\end{gathered}
$$

Using the matrix equation (5), we obtain

If

$$
\begin{aligned}
& \dot{V}(x(t))=-x^{T}(t) C x(t)+2 x^{T}(t)\left[-(A+B)^{T} H \Delta D\right. \\
& \left.+(\Delta A+\Delta B)^{T} H(E-D)-(\Delta A+\Delta B)^{T} H \Delta D\right] x(t) .
\end{aligned}
$$

$$
\lambda_{\min }(C)>2\left|(A+B)^{T} H \Delta D-(\Delta A+\Delta B)^{T} H(E-D)+(\Delta A+\Delta B)^{T} H \Delta D\right|
$$

then the total derivative of the Lyapunov function is negative definite for all matrices $\Delta D$, $\Delta A, \Delta B$, the elements of which are given as in (3).

On the basis of this theorem, it is possible to formulate more rigid, but easier to check, conditions of interval stability for the system (6).

Corollary: Let $(E-E)^{-1}(A+B)$ be asymptotically stable and there exist a positive definite matrix $H$, such that the inequality

$$
\lambda_{\min }\left[-(A+B)^{T} H(E-D)-(E-D)^{T} H(A+B)\right]
$$




$$
-2 \lambda_{\max }(H)[|A+B|\|\Delta D\|+\|\Delta A+\Delta B\|(|E-D|+\|\Delta D\|)]>0
$$

holds, then the system (6) is interval stable.

Using the obtained conditions of interval stability for the system without delay (6) consider the system (2). Denote a surface level of the Lyapunov function $V(x)=\alpha$ by $\partial V^{\alpha}$, and the region bounded by this surface by $V^{\alpha}$, that is

$$
\begin{aligned}
\partial V^{\alpha} & =\left\{x: x^{T}[(E-D)-\Delta D]^{T} H[(E-D)-\Delta D] x=\alpha\right\}, \\
V^{\alpha} & =\left\{x: x^{T}[(E-D)-\Delta D]^{T} H[(E-D)-\Delta D] x<\alpha\right\} .
\end{aligned}
$$

Lemma 1: Let $x(t)$ be a solution of (2) and for arbitrary $\alpha>0$ there exist $T>0$ such that $x(T) \in \partial V^{\alpha}$, and for $-\tau \leq s<T: x(s) \in V^{\alpha}$. Then for all $\theta:-\tau \leq \theta \leq T$, the following inequality holds true:

$$
|\dot{x}(\theta)|<\|\dot{x}(0)\|_{\tau}+\frac{\|A+\Delta A\|+\|B+\Delta B\|}{1-\|D+\Delta D\|} \sqrt{\varphi(H)}|x(T)|,
$$

where

$$
\varphi(H)=\frac{\lambda_{\max }\left([(E-D)-\Delta D]^{T} H[(E-D)-\Delta D]\right)}{\left.\lambda_{\min }[(E-D)-\Delta D]^{T} H[(E-D)-\Delta D]\right)} .
$$

Proof: Regarding the inequality of quadratic form and the conditions of Lemma 1, we have:

$$
\begin{gathered}
\left.\lambda_{\min }([E-D)-\Delta D]^{T} H[(E-D)-\Delta D]\right)|x(\theta)|^{2} \leq V(x(\theta)) \\
<V(x(T)) \leq \lambda_{\min }\left([(E-D)-\Delta D)^{T} H[(E-D)-\Delta D]\right)|x(T)|^{2} .
\end{gathered}
$$

That is why

$$
|x(\theta)|<\sqrt{\varphi(H)}|x(T)| .
$$

Regarding the form of system (2), conditions of Lemma 1 and the relation (10)

$$
\begin{gathered}
|\dot{x}(\theta)| \leq|D+\Delta D||\dot{x}(\theta-\tau)|+|A+\Delta A||x(\theta)|+|B+\Delta B||x(\theta-\tau)| \\
\quad<|D+\Delta D||\dot{x}(\theta-\tau)|+(|A+\Delta A|+|B+\Delta B|) \sqrt{\varphi(H)}|x(T)| .
\end{gathered}
$$

Constructing an analogous estimate for $\dot{x}(\theta-\tau)$, we obtain

$$
\begin{aligned}
|\dot{x}(\theta)|< & |D+\Delta D|^{2}|\dot{x}(\theta-2 \tau)| \\
& +(|A+\Delta A|+|B+\Delta B|)(1+|D+\Delta D|) \sqrt{\varphi(H)}|x(T)| .
\end{aligned}
$$

Let $\theta=n \tau+\tau_{1}$, where $0 \leq \tau_{1}<\tau, n \geq 1$. Then

$$
\begin{aligned}
|\dot{x}(\theta)|< & |D+\Delta D|^{n+1}|\dot{x}(\theta-(n+1) \tau)|+(|A+\Delta A|+|B+\Delta B|) \\
& \cdot\left(1+|D+\Delta D|+\ldots+|D+\Delta D|^{n}\right) \sqrt{\varphi(H)}|x(t)|
\end{aligned}
$$

Thus, $|D+\Delta D|<1$ and we have

$$
|\dot{x}(\theta)|<|\dot{x}(\theta-(n+1) \tau)|+\frac{|A+\Delta A|+|B+\Delta B|}{1-|D+\Delta D|} \sqrt{\varphi(H)}|x(T)| .
$$


Relation (9) follows here.

Theorem 2: Let $(E-D)^{-1}(A+B)$ be asymptotically stable and there exist a symmetric, positive definite matrix $H$ such that inequality

$$
\begin{gathered}
L(H)-2\left\|[(E-D)-\Delta D]^{T} H(B+\Delta B)\right\|(1+\sqrt{\varphi(H)}) \\
-4\left\|[(E-D)-\Delta D]^{T} H(D+\Delta D)\right\| \frac{\|A+\Delta D\|+\|B+\Delta B\|}{1-\|D+\Delta\|} \sqrt{\varphi(H)}|x(T)| .
\end{gathered}
$$

holds. Then system (2) is interval stable for arbitrary deviations $\tau>0$. Moreover, for arbitrary solutions $x(t)$ of the system (2), $|x(t)|_{1}<\epsilon$ for $t>0$ if $\|x(0)\|_{\tau}<\sigma_{1}(\epsilon)$ and $\|\dot{x}(0)\|_{\tau}<\sigma_{2}(\epsilon)$, where

$$
\begin{gathered}
\sigma_{1}(\epsilon)=\epsilon / \sqrt{\varphi(H)}, \\
\sigma_{2}(\epsilon)=\left[\frac{L(H)-2 \|[(E-D)-\Delta D)]^{T} H(B+\Delta B) \|(1+\sqrt{\varphi(H)})}{4\left\|[(E-D)-\Delta D]^{T} H(D+\Delta D)\right\|}\right. \\
\left.-\frac{\|A+\Delta A\|+\|B+\Delta\|}{1-\|D+\Delta D\|} \sqrt{\varphi(H)]}\right] \frac{\epsilon R}{\sqrt{\varphi(H)}} \\
R=\min \left\{1,\left[\frac{L(H)-2 \|[(E-D)-\Delta D)]^{T} H(B+\Delta B) \|(1+\sqrt{\varphi(H))}}{4\left\|[(E-D)-\Delta D]^{T} H(D+\Delta D)\right\|}\right.\right. \\
\left.\left.+(\sqrt{\varphi(H)}-1) \frac{\|A+\Delta A\|+\|B+\Delta B\|}{1-\|D+\Delta D\|}\right]^{-1}\right\} .
\end{gathered}
$$

Proof: Let $x(t)$ be a solution of the system (2) satisfying the condition $\|x(0)\|_{\tau}<\sigma_{1}(\epsilon)$. Then the solution $x(t),-\tau \leq t \leq 0$, is found inside the set $V^{\alpha}$, which is contained in the $R \epsilon$-neightborhood of the origin, if

$$
\alpha=(R \epsilon)^{2} \lambda_{\min }(H) .
$$

We show that this is prserved for arbitrary $t>0$. If not for arbitrary, some value $T>0$ such that $x(T) \in \partial V^{\alpha}$. Now consider the total derivative of the Lyapunov function (8) along the solution of the system

$$
\begin{gathered}
\dot{x}=[(E-D)-\Delta D]^{-1}[(A+B)+(\Delta A+\Delta B)] x(t)+[(E-D)-\Delta D]^{-1} \\
\cdot(D+\Delta D)[\dot{x}(t-\tau)-\dot{x}(t)]+[(E-D)-\Delta D]^{-1}(B+\Delta B)[x(t-\tau)-x(t)] .
\end{gathered}
$$

This system is obtained from (2) by means of the transformation:

$$
\begin{aligned}
\dot{V}(x(t))= & x^{T}(t)\left\{[(A+B)+(\Delta A+\Delta B)]^{T} H[(E-D)-\Delta D]+[(E-D)-\Delta D]^{T}\right. \\
& \cdot H[(A+B)+(\Delta A+\Delta B)]\} x(t)+2 x^{T}(t)[(E-D)-\Delta D]^{T} \\
& \cdot H\{(D+\Delta D)[\dot{x}(t-\tau)-\dot{x}(t)]+(B+\Delta B)[x(t-\tau)-x(t)]\} .
\end{aligned}
$$

Taking into account that the matrix $H$ satisfies equation (5), we derive 


$$
\begin{gathered}
\dot{V}(x(t)) \leq-\lambda_{\min }(C)|x(t)|^{2}+2 \mid(A+B)^{T} H \Delta D-(\Delta A+\Delta B)^{T} H(E-D) \\
+\left.(\Delta A+\Delta B)^{T} H \Delta D|| x(t)\right|^{2}+2\left|[(E-D)-\Delta D]^{T} H(D+\Delta D)\right| \\
\cdot|x(t)||\dot{x}(t)-\dot{x}(t-\tau)|+2\left|[(E-D)-\Delta D]^{T} H(B+\Delta B)\right||x(t)||x(t)-x(t-\tau)| . \text { Using }
\end{gathered}
$$

$L(H)$ as defined in (7), we have

$$
\begin{aligned}
\dot{V}(x(t)) \leq & -L(H)|x(t)|^{2} \\
& +2\left|[(E-D)-\Delta D]^{T} H(D+\Delta D)\right||\dot{x}(t)-\dot{x}(t-\tau)||x(t)| \\
& +2\left|[(E-D)-\Delta D]^{T} H(B+\Delta B)\right||x(t)-x(t-\tau)||x(t)| .
\end{aligned}
$$

According to the assumption, for $t=T$ we have $x(T) \in \partial V^{\alpha}$ and for $-\tau \leq s<T$ : $x(s) \in V^{\alpha}$. Therefore, using the inequality (10) and relations

$$
\begin{aligned}
& |x(t)-x(t-\tau)| \leq|x(t)|+|x(t-\tau)|, \\
& |\dot{x}(t)-\dot{x}(t-\tau)| \leq|\dot{x}(t)|+|\dot{x}(t-\tau)|,
\end{aligned}
$$

for $t=T$, we obtain

$$
\begin{gathered}
\dot{V}(x(T))<-L(H)|x(T)|^{2}+2\left|[(E-D)-\Delta D]^{T} H(D+\Delta D)\right||x(T)| \\
\cdot[|\dot{x}(T)|+|\dot{x}(T-\tau)|]+2\left|[(E-D)-\Delta D]^{T} H(B+\Delta B)\right|(1+\sqrt{\varphi(H)})|x(T)|^{2}
\end{gathered}
$$

Taking into account inequality (9) of Lemma 1 , we obtain

$$
\begin{gathered}
\dot{V}(x(T))<-\left[L(H)-2\left|[(E-D)-\Delta D]^{T} H(B+\Delta B)\right|(1+\sqrt{\varphi(H)})\right. \\
\left.-4\left|[(E-D)-\Delta D]^{T} H(D+\Delta D)\right| \frac{\|A+\Delta A\|+\|B+\Delta B\|}{1-\|D+\Delta D\|} \sqrt{\varphi(H)}\right]\|x(T)\|^{2} \\
+4\left|[(E-D)-\Delta D]^{T} H(D+\Delta D)\right||| \dot{x}(0)\left|{ }_{\tau}\right| x(T) \mid .
\end{gathered}
$$

If there exist a positive definite matrix $H$, satisfying inequality (11), then with

$$
\begin{gathered}
\|\dot{x}(0)\|_{\tau}<\left[\frac{L(H)-2\left\|[(E-D)-\Delta D]^{T} H(B+\Delta B)\right\|(1+\sqrt{\varphi(H)})}{4\left\|[(E-D)-\Delta D]^{T} H(D+\Delta D)\right\|}\right. \\
\left.-\frac{\|A+\Delta A\|+\|B+\Delta B\|}{1-\|D+\Delta D\|} \sqrt{\varphi(H)}\right] \frac{\epsilon R}{\sqrt{\varphi(H)}}, \quad R \leq 1
\end{gathered}
$$

the total derivative of the function $V(x(t))$ at $t=T$ is negative definite. This provides $x(t) \in V^{\alpha}$ for all $t>0$.

As it follows from Lemma 1, in this case

$$
\begin{aligned}
|\dot{x}(t)| & <\left[\frac{L(H)-2\left\|[(E-D)-\Delta D]^{T} H(B+\Delta B)\right\|(1+\sqrt{\varphi(H)})}{4\left\|[(E-D)-\Delta D]^{T} H(D+\Delta D)\right\|}\right. \\
& \left.-\frac{\|A+\Delta A\|+\|B+\Delta B\|}{1-\|D+\Delta D\|} \sqrt{\varphi(H)}\right] \frac{\epsilon R}{\sqrt{\varphi(H)}}
\end{aligned}
$$




$$
+\frac{\|A+\Delta A\|+\|B+\Delta B\|}{1-\|D+\Delta D\|} \sqrt{\varphi(H)} \epsilon R
$$

In order that $|\dot{x}(t)|<\epsilon$ holds true for $t>0$, it suffices to set variable according to (12).

The sufficient condition given by inequality (11) imposes rigid restrictions on th system parameters and their admissible perturbations. It is possible to weaken the assumptions of Theorem 1 and obtain conditions of interval stability, depending on the size of delay.

Lemma 2: Let $x(t)$ be a solution of the system (2) such that $\|x(0)\|{ }_{\tau}<\sigma$. Then, for $0<t \leq \tau$, the following inequality holds:

$$
|x(t)|<(1+2\|D+\Delta D\|+\|B+\Delta B\| \tau) \sigma e^{\|A+\Delta A\| \tau} .
$$

Proof: Write the system (2) in the form

$$
\begin{aligned}
& x(t)=x(0)+(D+\Delta D)[x(t-\tau)-x(-\tau)] \\
& +\int_{0}^{t}[(A+\Delta A) x(s)+(B+\Delta B) x(s-\tau)] d s .
\end{aligned}
$$

Under $0<t \leq \tau$, the relation

$$
x(t)<\sigma+2|D+\Delta D| \sigma+|A+\Delta A| \int_{0}^{t}|x(s)| d s+|B+\Delta B| \sigma \tau
$$

holds. Using the inequality of Gronwall-Bellman, we derive

$$
|x(t)|<(1+2|D+\Delta D|+|B+\Delta B| \tau) \sigma \epsilon^{|A+\Delta A| \tau} .
$$

From here it follows (13).

Lemma 3: Let $x(t)$ be a solution of system (2) and for an arbitrary $\alpha>0$ there exist $t>\tau$ such that $x(s) \in V^{\alpha}$ for $-\tau \leq s<T$ and $x(t) \in \partial V^{\alpha}$ for $s=T$. Then the following equality holds:

$$
\begin{aligned}
|x(T)-x(T-\tau)|< & \|D+\Delta D\|\|x(0)\|_{\tau}+\frac{\|A+\Delta A\|+\|B+\Delta B\|}{1-\|D+\Delta D\|} \\
& \cdot \sqrt{\varphi(H)} \tau|x(T)|
\end{aligned}
$$

Proof: Write the system (2) in the following form

$$
\begin{gathered}
x(T)=x(T-\tau)+(D+\Delta D)[x(T-\tau)-x(T-2 \tau)] \\
\quad+\int_{T-\tau}^{T}[(A+\Delta A) x(s)+(B+\Delta B) x(s-\tau)] d s .
\end{gathered}
$$

Taking into consideration conditions of Lemma 3, we obtain

$$
\begin{gathered}
|x(T)-x(T-\tau)| \leq|D+\Delta D||x(T-\tau)-x(T-2 \tau)| \\
+[|A+\Delta A|+|B+\Delta B|] \sqrt{\varphi(H)} \tau|x(T)| .
\end{gathered}
$$

Constructing an analogous estimate for the expression $|x(T)-x(T-2 \tau)|$ : 


$$
\begin{gathered}
|x(T)-x(T-\tau)| \leq|D+\Delta D|^{2}|x(T-2 \tau)-x(T-3 \tau)|+(1+|D+\Delta D|) \\
\cdot[|A+\Delta A|+|B+\Delta B|] \sqrt{\varphi(H)} \tau|x(T)| .
\end{gathered}
$$

Let $T=n \tau+\tau_{1}$ for $0 \leq \tau_{1}<\tau$ and $n \geq 1$. Continuing the iterative process, we obtain

$$
\begin{aligned}
& |x(T)-x(T-\tau)| \leq|D+\Delta D|^{n}[|x(T-n \tau)-x(0)|-|x(0)-x(T-(n+1) \tau)|] \\
& +\left[1+|D+\Delta D|+\ldots+|D+\Delta D|^{n-1}[|A+\Delta A|+|B+\Delta B|] \sqrt{\varphi(H)} \tau|x(t)| .\right.
\end{aligned}
$$

So long as $|D+\Delta D|$, we get (14).

Lemma 4: Let $x(t)$ be a solution of the system (2) for an arbitrary $\alpha>0$ there exist $T>0$ such that $x(s) \in V^{\alpha}$ for $-\tau \leq S<T$ and $x(T) \in \partial V^{\alpha}$ for $s=T$. Then the estimate

$$
\begin{aligned}
&|\dot{x}(T)-\dot{x}(T-\tau)|<4\|D+\Delta D\|\left[\|\dot{x}(0)\|_{\tau}+\frac{\|A+\Delta A\|+\|B+\Delta B\|}{1-\|D+\Delta D\|}\|x(0)\|_{\tau}\right] \\
&+\left(\frac{\|A+\Delta A\|+\|B+\Delta B\|}{1-\|D+\Delta D\|}\right)^{2} \sqrt{\varphi(H)} \tau|x(T)|
\end{aligned}
$$

holds.

Proof: Regarding the form of the system (2), we have:

$$
\begin{gathered}
|\dot{x}(T)-\dot{x}(T-\tau)|<|D+\Delta||x(T-\tau)-\dot{x}(T-2 \tau)| \\
+(|A+\Delta A|+|B+\Delta B|) \\
\cdot\left[4\|D+\Delta D\|\|x(0)\|_{\tau}+\frac{\|A+\Delta A\|+\|B+\Delta B\|}{1-\|D+\Delta D\|} \sqrt{\varphi(H)} \tau|x(T)|\right] .
\end{gathered}
$$

Continuing, we obtain

$$
\begin{gathered}
|\dot{x}(T)-\dot{x}(T-\tau)| \leq|D+\Delta D|^{n}[|\dot{x}(T-n \tau)-\dot{x}(0)|+|\dot{x}(0)-\dot{x}(T(n+1) \tau)|] \\
+\left[1+|D+\Delta D|+\ldots+|D+\Delta D|^{n-1}\right](|A+\Delta A|+\|B+\Delta B\|) \\
\cdot\left[4\|D+\Delta D\|\|x(0)\|_{\tau}+\frac{\|A+\Delta A\|+\|B+\Delta B\|}{1-\|D+\Delta D\|} \sqrt{\varphi(H)} \tau|x(T)|\right] .
\end{gathered}
$$

That follows

$$
\begin{aligned}
& |\dot{x}(T)-\dot{x}(T-\tau)| \leq 4|D+\Delta D|\|\dot{x}(0)\|_{\tau}+\frac{|A+\Delta A|+|B+\Delta B|}{1-|D+\Delta D|} \\
& \cdot\left[4\|D+\Delta D\|\|x(0)\|_{\tau}+\frac{\|A+\Delta A\|+\|B+\Delta B\|}{1-\|D+\Delta D\|} \sqrt{\varphi(H)} \tau|x(T)|\right] .
\end{aligned}
$$

We obtain inequality (15).

With the given lemmas, we obtain the conditions of system (2) interval stability which depends on argument deviation. 
Theorem 3: Let $(E-D)^{-1}(A+B)$ be asymptotically stable matrix and condition (7) holds. Then with $\tau<\tau_{0}$, where

$$
\begin{aligned}
\tau_{0}= & \frac{L(H)(1-\|D+\Delta D\|)}{2(\|A+\Delta A\|+\|B+\Delta B\|) \sqrt{\varphi(H)}}\left[\left\|[(E-D)-\Delta D]^{T} H(B+\Delta B)\right\|\right. \\
& \left.+\left\|[(E-D)-\Delta D]^{T} H(D+\Delta D)\right\| \frac{\|A+\Delta A\|+\|B+\Delta B\|}{1-\|D+\Delta D\|}\right]^{-1}
\end{aligned}
$$

system (2) be intervally stable. And also, for arbitrary solution $x(t)$ holds $|x(t)|_{1}<\epsilon$, $t>0$ if $\|x(0)\|_{\tau}<\sigma_{1}(\epsilon, \tau)$ and $\|\dot{x}(0)\|_{\tau}<\sigma_{2}(\epsilon, \tau)$ where

$$
\begin{gathered}
\sigma_{1}(\epsilon, \tau)=\min \left\{\frac{e^{-\|A+\Delta A\| \tau}}{1+2\|D+\Delta D\|+\|B+\Delta B\| \tau}, \frac{L(H)\left(1-\tau / \tau_{0}\right) \zeta}{8\|D+\Delta D\|}\right. \\
{\left[[(E-E)-\Delta D]^{T} H(B+\Delta B)\|+\|[(E-D)-\Delta D]^{T} H(D+\Delta D) \|\right.} \\
\left.\left.. \frac{\|A+\Delta A\|+\|B+\Delta B\|}{1-\|D+\Delta D\|}\right]\right\} \frac{R \epsilon}{\sqrt{\varphi(H)}}, \\
\sigma_{2}(\epsilon, \tau)=\frac{L(H)\left(1-\tau / \tau_{0}\right)(1-\zeta)}{8\|D+\Delta D\| \cdot\left\|[(E-D)-\Delta D]^{T} H(D+\Delta D)\right\|} \frac{R \epsilon}{\sqrt{\varphi(H)}}, \\
R=\min \left\{1,\left[\frac{L(H)\left(1-\tau / \tau_{0}\right)(1-\zeta)}{8\|D+\Delta D\| \cdot\left\|[(E-D)-\Delta D]^{T} H(D+\Delta D)\right\| \sqrt{\varphi(H)}}\right.\right. \\
\left.\left.+\frac{\|A+\Delta A\|+\|B+\Delta B\|}{1-\|D+\Delta D\|} \sqrt{\varphi(H)}\right]^{-1}\right\},
\end{gathered}
$$

and $0<\zeta<1$ is arbitrary constant.

Proof: Let $x(t)$ be a solution of system satisfying the condition $\|x(0)\|_{\tau}<\sigma_{1}(\epsilon, \tau)$. Then, as follows from inequality (13) of Lemma 2, for arbitrary $\epsilon>0, \tau>0$, the solution $x(t)$ for $0 \leq t \leq \tau$ is contained by set $V^{\alpha}, \alpha=(R \epsilon)^{2} \lambda_{\min }(H)$, which is contained in $R \varepsilon$ vicinity of coordinates origin. Show the same for $t>T$. So by contradiction, exist $T>\tau$, that $x(t) \in V^{\alpha}$ holds. Estimate the total derivative of function $V(x)(8)$ along the solution $x(t)$ of system (2). If transformations of Theorem 2 are repeated, we obtain

$$
\begin{gathered}
\left.\dot{V}(x(t)) \leq-L(H)\left|x(t)^{2}+2\right|[(E-D)-\Delta D]^{T} H(D+\Delta D)\right\} \\
\cdot|x(t)| \cdot|\dot{x}(t)-\dot{x}(t-\tau)| \\
+2\left|[(E-D)-\Delta D]^{T} H(B+\Delta B)\right| \cdot|x(t)| \cdot|x(t)-x(t-\tau)| .
\end{gathered}
$$

Along the assumption, for $t=T$ the right outcome of solution $x(t)$ to level $V^{\alpha}$ surface takes place. With inequality (14) and (15) by Lemmas 3 and 4 we obtain

$$
\dot{V}(x(T))<-L(H)|x(T)|^{2}+2\left|[(E-D)-\Delta D]^{T} H(B+\Delta B)\right|
$$




$$
\begin{gathered}
\cdot\left\{4\|D+\Delta D\|\|x(0)\|_{\tau}+\frac{\|A+\Delta A\|+\|B+\Delta B\|}{1-\|D+\Delta D\|} \sqrt{\varphi(H)} \tau|x(T)|\right\}|x(T)| \\
+2\left|[(E-D)-\Delta D]^{T} H(D+\Delta D)\right| \\
\cdot\left\{4 \| D+\Delta D \mid\left[\|\dot{x}(0)\|_{\tau}+\frac{\|A+\Delta A\|+\|B+\Delta B\|}{1-\|D+\Delta D\|}\|x(0)\|_{\tau}\right]\right. \\
\left.+\left[\frac{\|A+\Delta A\|+\|B+\Delta B\|}{1-\|D+\Delta D\|}\right]^{2} \sqrt{\varphi(H)} \tau|x(T)|\right\}|x(T)|
\end{gathered}
$$

The right-hand side of the transformation gives

$$
\begin{gathered}
\dot{V}(x(T))<-\left\{L(H)-2 \frac{\|A+\Delta A\|+\|B+\Delta B\|}{1-\|D+\Delta D\|}\left[\mid[(E-D)-\Delta D]^{T} H\right.\right. \\
\left.\cdot(B+\Delta B)|+|[(E-D)-\Delta D]^{T} H(D+\Delta D) \mid \frac{\|A+\Delta A\|+\|B+\Delta B\|}{1-\|D-\Delta D\|}\right] . \\
\cdot \sqrt{\varphi(H)} \tau\}|x(T)|^{2}+8\|D+\Delta D\|\left[\left|[(E-D)-\Delta D]^{T} H(B+\Delta B)\right|\right. \\
\left.\quad+\left|[(E-D)-\Delta D]^{T} H(D+\Delta D)\right| \frac{\|A+\Delta A\|+\|B+\Delta B\|}{1-\|D+\Delta D\|}\right]\|x(0)\|_{\tau} \\
+8\|D+\Delta D\|\left|[(E-D)-\Delta D]^{T} H(D+\Delta D)\right|\|\dot{x}(0)\| \tau .
\end{gathered}
$$

Let us set

$$
\begin{aligned}
\tau_{0}= & \frac{L(H)(1-\|D+\Delta D\|)}{2(\|A+\Delta A\|+\mid B+\Delta B \|) \sqrt{\varphi(H)}}\left[\left\|[(E-D)-\Delta D]^{T} H(B+\Delta B)\right\|\right. \\
& \left.+\|[(E-D)-\Delta D]^{T} H(D+\Delta D) \frac{\|A+\Delta A\|+\|B+\Delta B\|}{1-\|D+\Delta D\|}\right]^{-1}
\end{aligned}
$$

and designate $\xi=\tau / \tau_{0}$. For the estimation of total Lyapunov's function derivative in time $t=T$ the following inequality holds:

$$
\begin{aligned}
\dot{V}(x(T))< & -L(H)(1-\xi)|x(T)|^{2}+8\|D+\Delta D\|\left[\mid[(E-D)-\Delta D]^{T} H(B+\Delta B)\right. \\
& \left.+\left|[(E-D)-\Delta D]^{T} H(D+\Delta D)\right| \frac{\|A+\Delta A\|+\|B+\Delta B\|}{1-\|D+\Delta D\|}\right]\|x(0)\|_{\tau} \\
& +8\|D+\Delta D\|\left|[(E-D)-\Delta D]^{T} H(D+\Delta D)\right|\|\dot{x}(0)\|_{\tau} .
\end{aligned}
$$

Let

$$
\begin{gathered}
\left.\sigma_{1} \leq \frac{L(H)(1-\xi) \zeta}{8\|D+\Delta D\|}\left[\left|[(E-D)-\Delta D]^{T} H(B+\Delta B)\right|+\mid\right](E-D)-\Delta D\right]^{T} \\
\left.\cdot H(D+\Delta D) \frac{\|A+\Delta A\|+\|B+\Delta B\|}{1-\|D+\Delta D\|}\right]^{-1} \frac{R \epsilon}{\sqrt{\varphi(H)}} \\
\sigma_{2} \leq \frac{L(H)(1-\xi)(1-\zeta)}{8\|D+\Delta D\| \|[(E-D)-\Delta D]^{T} H(D+\Delta D) \mid} \frac{R \epsilon}{\sqrt{\varphi(H)}}
\end{gathered}
$$

is proposed with $0<\zeta<1$-arbitrary constant. Then $\dot{V}(x(T))$ is negative defined and $x(t) \in V^{\alpha}$ holds for all $t>0$. Let us estimate the value $|\dot{x}(t)|$. Inequalities (9) and (18) give us that if $x(t) \in V^{\alpha}$, then 


$$
\begin{aligned}
|\dot{x}(t)| & <\frac{L(H)(1-\xi)(1-\zeta)}{8\|D+\Delta D\| \|[(E-D)-\Delta D]^{T} H(D+\Delta D) \mid} \frac{R \epsilon}{\sqrt{\varphi(H)}} \\
& +\frac{\|A+\Delta A\|+\|B+\Delta B\|}{1-\|D+\Delta D\|} \sqrt{\varphi(H)} R \epsilon
\end{aligned}
$$

holds. Therefore, in order for $|\dot{x}(t)|<\epsilon, t>0$, the inequality

$$
\begin{aligned}
R \leq & {\left[\frac{L(H)(1-\xi)(1-\zeta)}{8\|D+\Delta D\| \|[(E-D)-\Delta D]^{T} H(D+\Delta D) \mid \sqrt{\varphi(H)}}\right.} \\
& \left.+\frac{\|A+\Delta A\|+\|B+\Delta B\|}{1-\|D+\Delta D\|} \sqrt{\varphi(H)}\right]^{-1}
\end{aligned}
$$

is sufficient. That allows us to obtain (17).

\section{Acknowledgement}

I would like to acknowledge the support of Professor A.A. Martynyuk for his help and encouragement regarding the compilation of this working paper.

\section{References}

[1] Andreeva, E., Kolmanovsij, V., and Shaikhet, L., Control of Hereditary Systems, Nauka, Moscow 1992.

[2] Bellman, R. and Cooke, K., Differential-Difference Equations, Math. in Science and Engineering, New York, London 1963.

[3] Hale, J.K., Verduyn Lunel, S.M., Introduction to Functional Differential Equations, Springer-Verlag, New York 1993.

[4] Jabko, A.P. and Kharitonov, V.L., Quasipolynomial class stability, Avtomatika 2 (1992), 3-14.

[5] Jabko, A.P. and Kharitonov, V.L., Quasipolynomial of neutral type class stability, Avtomatika i Telemechanika 1 (1993), 92-106.

[6] Khusainov, D.Ya. and Mustaphaeva, R., Robust stability of systems with delay, Ukrain. Matem. Zhurn. 47:6 (1995), 859-863.

[7] Khusainov, D.Ya. and Yun'kova, E.A., Investigation of the stability of linear systems of neutral type to be the Lyapunov function methods, Diff. Urav. 24:4 (1988), 613-621.

[8] Lakshmikantham, V. and Martynyuk, A.A., The development of direct Lyapunov method for the systems with delay (Review), Pricladnaya Mech. 29:2 (1993), 3-16.

[9] Pinney, E., Ordinary Difference Equations, University of California Press, Berkeley and Los Angeles 1958.

[10] Shyn, K.K. and Van, J.J., Robust stability of uncertain time-delay systems and its stabilization by variable structure control, Int. J. Contr. 57:1 (1993), 237-246. 


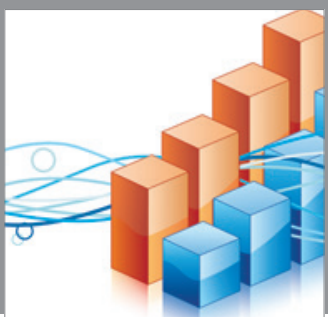

Advances in

Operations Research

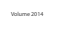

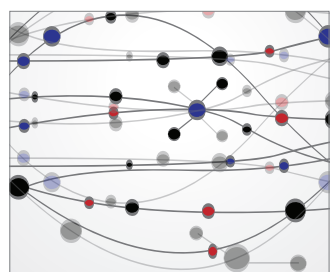

\section{The Scientific} World Journal
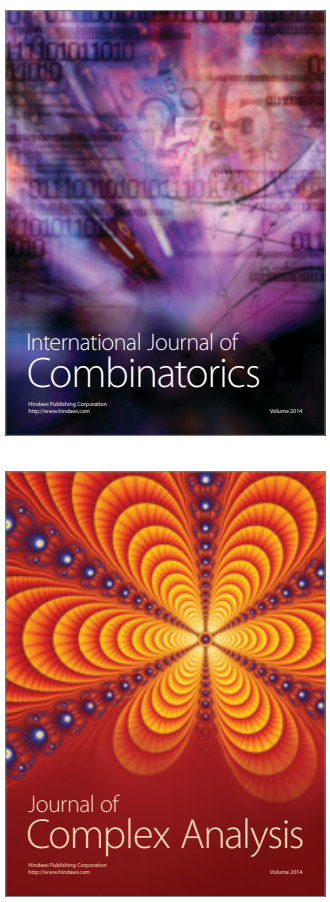

International Journal of

Mathematics and

Mathematical

Sciences
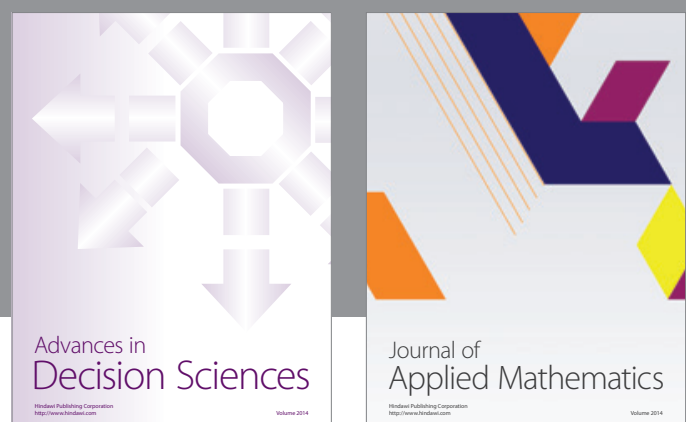

Journal of

Applied Mathematics
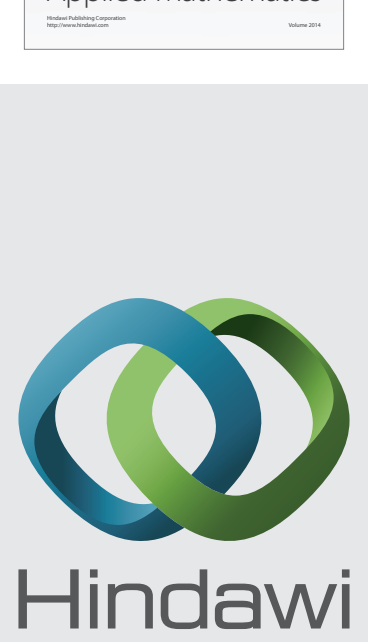

Submit your manuscripts at http://www.hindawi.com
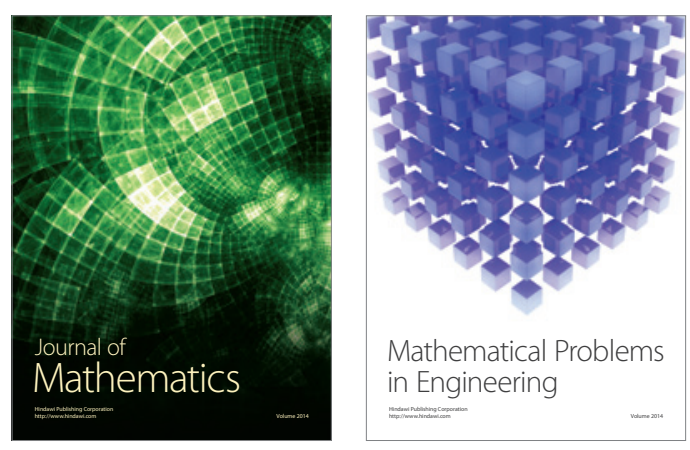

Mathematical Problems in Engineering
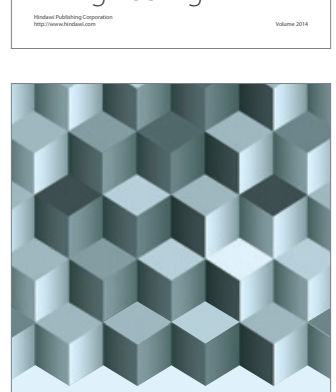

Journal of

Function Spaces
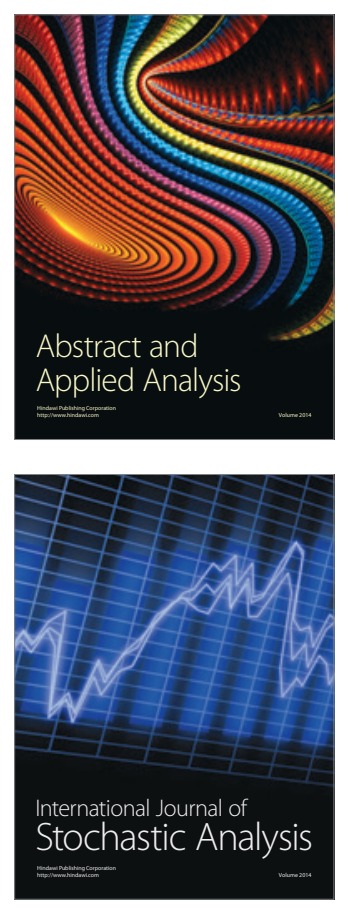

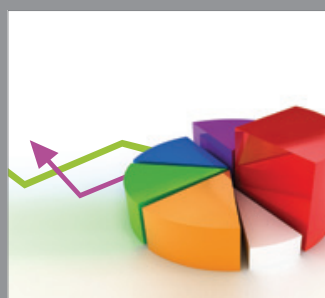

ournal of

Probability and Statistics

Promensencen
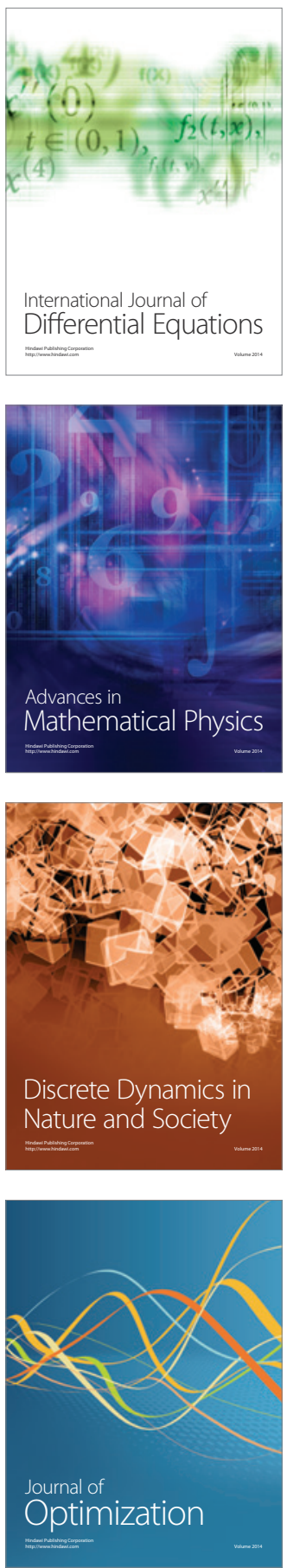